\title{
Advances in the Management of Unresectable or Metastatic Pancreatic Neuroendocrine Tumors: Chemotherapy, Targeted Therapy, Hormonal Treatment, and Future Directions
}

\begin{abstract}
Ahmet Bilici
Abstract

Pancreatic neuroendocrine tumors (pNETs) are rare and heterogenous tumors and surgery to remove the primary tumor is the mainstay of treatment for resectable disease. However, curative surgery is often not feasible, because half of patients with pNET have metastases at the time of diagnosis. Palliative dubulking surgery and liver-directed therapies are appropriate options for these patients. Streptozocin-based regimens are standard, although temozolamide-based treatments are rapidly gaining wide clinical application. Somatostatin analogs are mainly indicated in hormonally active tumors to ameliorate symptoms. In addition, anti-tumoral activity has been proven in well-differentiated NETs. Recently, there has been tremendous progress in the molecular biology of pNETs; thereby, the efficacy of sunitinib and everolimus in the treatment of patients with metastatic pNETs has been proven by large placebo-controlled phase III trials. Currently, there are no definitively proven predictive biomarkers to evaluate response to medical therapies in patients with pNET. Therefore, further studies are needed to individualize and optimize their management. This article reviews systemic chemotherapy, targeted therapies, and anti-secretory treatments for the management of patients with unresectable or metastatic pNETs, summarized in the light of recent advances.
\end{abstract}

Keywords: Neuroendocrine tumors - pancreatic - chemotherapy - targeted therapy - somatostatin analogues

Asian Pac J Cancer Prev, 16 (6), 2151-2159

\section{Introduction}

Gastroenteropancreatic neuroendocrine tumors (NETs) originate from neuroendocrine cells in the islets of Langerhans cells of the pancreas (pNET) or enterochromaffin cells of digestive tract or thorax (Strosberg et al., 2008; Yao et al., 2008; Zeng et al., 2013). These tumors are rare disease, but the incidence and prevelance of NETs seem to be increasing based on the most recent US Surveillance Epidemiology and End Results (Yao et al., 2008; Niederle et al., 2010). The majority of them are sporadic, however, they may arise as a result of familiar syndromes such as MEN 1 syndrome, von Hippel-Lindau disease, and neurofibromatosis type 1. NETs have different and complex clinical behavior and their clinical characteristics depend on the site of the primary tumor and its ability to secrete hormones and bioamines. Thus, NETs can be divided into two groups: functional and non-functional (Halfdanarson et al., 2008). Somatostatin analogs are effectively used to inhibit hormonal secretion and improve symptoms in about $75 \%$ of patients with carcinoid syndrome (Modlin et al., 2010).

The majority of patients with NETs are developed at the time of diagnosis and liver is the predominant localization in most cases. Therefore, histologic differentiation, proliferative activity, and stage are the strongest predictors of survival. In patients with localized-well-differentiated NETs, five-year survival is $60-100 \%$, while patients with well- and moderately differentiated distant metastases have a five-year survival probability of $35 \%$. This is less than $5 \%$ in poorly differentiated metastatic NET (Yao et al., 2008; Pavel et al., 2012).

pNETs are also rare tumors and surgery to remove the primary tumor is mainstay of treatment for resectable disease and currently the only curative option. However, it is dependent on the stage of disease. Curative surgery is often not feasible, because half of patients with pNET have metastatic disease at the time of diagnosis (Ballian et al., 2009; Pavel et al., 2012; Ramage et al., 2012). In these patients, palliative dubulking surgery for primary tumor and therapy directed to the liver are recommended (Yao et al., 2001; Knigge et al., 2008). Streptozocin-based regimen remains the standard regimen with 7-20 months of time to progression (Eriksson et al., 1990; Delaunoit et al., 2004; Kouvaraki et al., 2004; Sun et al., 2005), but recently temozolamide-based regimen has rapidly gained in wide clinical application with an impressive response rate of $70 \%$ (Strosberg et al., 2011).

Advances in the understanding of the molecular biology of pNETs has revealed increased expression 
of several cellular growth factors and their receptors (including vascular endothelial growth factor [VEGF] and the VEGF receptor [VEGFR]), and involvement of the mammalian Target of Rapamycin (mTOR) in pancreatic neuroendocrine tumorigenesis (Missiaglia et al., 2010; Oberg et al., 2013; Zhang et al., 2013). Recently, the efficacy of everolimus and sunitinib with improved time to disease progression has been proved by two phase III pivotal studies and approved by the Food and Drug Administration (FDA) in patients with pNET (Yao et al., 2011; Raymond et al., 2011). Despite these marked advances, the prognosis of patients with advanced pNET remains poor and new therapeutic molecular targets are required to improve survival outcomes. This review discusses the currently available management options in light of the most recent literatures and guidelines, along with promising therapeutic options that are still under development for patients with pNET.

\section{Current Systemic Treatment Modalities for Advanced pNET}

\section{Chemotherapy}

Systemic chemotherapy has been comprehensively investigated and considered to be effective options for patients with progressive pNET compared to those with non-pNET. Especially well-differentiated pNETs respond well to cytotoxic chemotherapy. Therefore, for patients who are highly symptomatic from tumor bulk or have rapidly progressing metastases, cytotoxic chemotherapy rather than molecularly targeted therapy or somatostatin analogs are recommended because of the higher objective response rate (RR) (Pavel et al., 2012; Khagi and Saif, 2014; Singh et al., 2014). Alkylating agents, pyrimidine analogs, anthracyclines, and platinum compounds have previously been studied, and streptozocin and temozolamide have been found to be paticularly active in pNETs. However, platin-based chemotherapy combinations have been used for G3 and non-pNETs (Pavel et al., 2012; Singh et al., 2014).

Streptozocin-based combination therapy has been an historical treatment standard for patients with advanced pNETs since the 1980s (Khagi and Saif, 2014). In an early randomized trial performed by Moertel et al. (1992) streptozocin plus doxorubicin was compared with streptozocin and 5-fluorouracil (5-FU) in 105 patients with advanced pNET. The authors showed that streptozocin plus doxorubicin had a combined biochemical and radiologic RR of $69 \%$ and a superior median survival (2.2 vs 1.4 years). Kouvaraki et al. (2004) in their retrospective analysis of 84 patients with either locally advanced or metastatic pNETs treated with streptozocin, 5-FU, and doxorubicin, reported a $39 \%$ objective radiographic $\mathrm{RR}$ and a median survival duration of 37 months. On the other hand, although streptozocin-based regimens are clearly active in patients with advanced pNET, the widespread usage has been limited because of toxicity, which can include nausea, hair loss, renal dysfunction, and hematologic toxicity (Khagi and Saif, 2014).

Dacarbazine is an alkylating agent that has activity against pNET. In a phase II trial, 42 patients with advanced pancreatic islet cell carcinomas were treated with dacarbazine and the objective response rate was found to be $33 \%$ (Ramanathan et al., 2001). However, the toxicity of dacarbazine-based regimens has limited their widespread use as streptozocin. Temozolomide is an alkylating agent and orally active analog of dacarbazine with activity in pNETs. Retrospective series and a preliminary report of a prospective phase II trial have also reported promising activity with temozolomide-based cytotoxic regimens in patients with pNET. In prospective studies, temozolomide-based combinations have provided 24 to $45 \%$ of overall RR (Kulke et al., 2006; Chan et al., 2012; Chan et al., 2013). Strosberg et al. in their single-arm retrospective study investigated the efficacy of oral temozolamide $\left(200 \mathrm{mg} / \mathrm{m}^{2}\right.$ daily on days 10 to 14) plus capecitabine $\left(750 \mathrm{mg} / \mathrm{m}^{2}\right.$ twice daily on days 1 to 14 ) therapy, and they indicated that objective RR was $70 \%$ with a median progression-free survival (PFS) of 18 months and overall survival (OS) of $92 \%$ at two years (Strosberg et al., 2011). A preliminary report of a prospective phase II trial was presented at the 2014 ASCO Gastrointestinal Cancers Symposium. In this study the use of combination therapy with capecitabine (CAPTEM) was evaluated in gastroenteropancreatic NET. It showed that an objective partial response was obtained in 4 of 11 patients with advanced pNET $(36 \%)$ with a median PFS of $>20$ months (Fine et al., 2014). A phase III trial evaluating the relative efficacy of the temozolomide plus capecitabine versus temozolomide alone for patients with advanced pNET is ongoing (NCT01824875). In patients with glioblastoma the relationship between expression of methylguanine DNA methyltransferase (MGMT) and temozolomide responsiveness has been investigated. In one study, the prevalence of MGMT deficiency was significantly higher in pNETs than in carcinoid tumors. Of 21 patients treated with temozolomide-based regimens, none of the 16 with intact MGMT expression (including all 13 carcinoid tumors) responded to treatment. In contrast, 4 of 5 patients whose tumors lacked MGMT expression (all pNETs) had a radiologic response. In spite of these compelling results, routine testing of MGMT expression to select patients for temozolomide therapy is not yet a standard approach, but it should be considered to be a potential biomarker that may influence repponse to treatment (Kulke et al., 2009).

Platinum-based combinations have been used as a standard first-line option for patients with poorlydifferentiated or anaplastic pNET (G3 or high-grade). In particular, etoposide in combination with cisplatin (EP) is considered as the standard care of patients with highgrade pNET, with $41.5 \%$ of objective RR and 9.2 months of median duration of response (Mitry et. al.,1999). Several other regimens including paclitaxel-carboplatinetoposide, capecitabine-oxaliplatin, and carboplatinvicristine-etoposide have been previously investigated, but they were not indicated to be superior to the standard EP regimen (Hainsworth et al., 2006; Bajetta et al., 2007; Olsen et al., 2012).

Preliminary results of some studies demonstrated that oxaliplatin had antitumor activity for patients with pNETs in combination with 5-FU, capecitabine and 
Table 1. Selected Clinical Trials of Systemic Chemotherapy in Patients with Advanced Pancreatic Neuroendocrine Tumors

\begin{tabular}{llccl}
\hline Regimen & \multicolumn{3}{c}{ No. patients Response rate, $\%$} & Median PFS and OS, months \\
\hline Moertel et al. & STZ-DOX vs STX-5-FU vs Chloro & 105 & 69 vs 45 vs 30 & PFS, 18 vs 14 vs 17; OS, 26.4 vs 16.8 vs 18 \\
Kouvaraki et al. & STZ-5-FU-DOX & 84 & 39 & PFS, 18;OS, 37 \\
Ramanathan et al. & DTIC & 42 & 33 & PFS, NR; OS, 19.3 \\
Strosberg et al. & TEM-CAP & 30 & 70 & PFS, 18; OS, NR \\
Kulke et al. & TMZ-TALD & 11 & 45 & PFS, NR; OS, NR \\
Chan et al. & TMZ-BEV & 15 & 33 & PFS, 14.3; OS, 41.7 \\
Fine et al. & TMZ-CAP & 11 & 36 & PFS, >20; OS, >24.4 \\
\hline
\end{tabular}

STZ, streptozocin; DOX, doxorubicin; 5-FU, 5- fluorouracil; Chloro, chlorozotocin; DTIC, dacarbazine; CAP, capecitabine; TALD, thalidomide; $\mathrm{BEV}$, bevacizumab; PFSm progression-free survival; OSm overall survival; NR, not reached

bevacizumab (Bajetta et al., 2007; Venook et al., 2008; Kunz et al., 2010). A phase II trial carried out by Kunz et al. evaluated an activity for capecitabine in combination with oxaliplatin and bevacizumab in patients with advanced NET. Preliminary report showed that 7 of 31 evaluable patients $(23 \%)$ had a partial response; 22 others had stable disease $(71 \%)$ with median PFS reported to be 13.2 months (Kunz et al., 2010). Bajetta et al. (2007) indicated similar outcomes with capecitabine plus oxaliplatin alone. Table 1 shows selected clinical trials of systemic chemotherapy in patients with advanced pNET.

\section{Targeted treatments}

Recent studies related to the molecular biology of pNETs has proved elevated expression of several cellular growth factors and their receptors in pancreatic neuroendocrine tumorigenesis (Missiaglia et al., 2010; Oberg et al., 2013; Zhang et al., 2013). They have particularly focused on the role of VEGF and mTOR pathways. mTOR is a central regulator of protein synthesis important in cancer, including cell growth and proliferation, angiogenesis, and cell metabolism (Tokunaga et al., 2004). Recently, trials associated with targeted agents have indicated antitumor activity associated with bevacizumab and several tyrosine kinase (TK) inhibitors that inhibit VEGFR, as well as the mTOR inhibitor everolimus (Kulke et al., 2008; Yao et al., 2008; Yao et al., 2010; Castellano et al., 2013). Moreover, these agents seem to be more effective in pNETs than in advanced gastrointestinal NETs.

\section{mTOR inhibitors}

One of the important potential targets is the mTOR pathway in pNETs. mTOR is a serine/threonine protein kinase that regulates cell metabolism, cell proliferation, and protein synthesis (Tokunaga et al., 2004). It has been demonstrated to have an important role in many of the genetic syndromes including tuberous sclerosis and neurofibramatosis 1 , which are associated with increased propensity of pNET (Jensen et al., 2008). Everolimus (RAD001), inhibitor of mTOR, results in a decrease in insülin-like growth factor 1 receptor and NET cell growth in studies on pancreatic neuroendocrine cell lines (Zitzmann et al., 2007).

Firstly, nonrandomized studies have investigated the activity of everolimus, with and without octreotide. An international multicenter phase II trial (RADIANT-1) initially explored the activity of everolimus (10 mg daily) in 160 patients with pNET. Forty-five of them were also administered concurrent octreotide at the discretion of the investigators (Yao et al., 2010). The median PFS time for patients who received octreotide plus everolimus was better than that of patients receiving everolimus only (17 vs 9.7 months), but whether octreotide contributed to the higher PFS in this cohort is not clear, because the study was not randomized.

The placebo-controlled, double-blind, and randomized phase III RADIANT-3 trial evaluated the activity of everolimus monotherapy (10 mg daily) plus best supportive care (BSC) compared with placebo and BSC in 410 patients with advanced progressing pNET (Yao et al., 2011). The majority of the patients had welldifferentiated or moderately differentiated tumors and half of the patients in each arm had previously been treated with chemotherapy and $50 \%$ of the patients in each arm also received somatostatin analogs. Results indicated that everolimus was associated with a significant prolongation in median PFS (11.0 vs 4.6 months), which resulted in a $65 \%$ reduction in the estimated risk of progression $(\mathrm{p}<0.001)$. The benefit from everolimus in this cohort appeared to be primarily stable disease and minör tumor shrinkage, but the duration of therapy was significantly longer in the everolimus arm. However, there was no OS benefit between the two arms $(\mathrm{p}=0.594)$. Drugrelated adverse events (everolimus vs placebo group) were mostly grade 1 or 2 , and included stomatitis (64 vs 17\%, respectively), rash (49 vs 10\%), diarrhea (34 vs $10 \%$ ), fatigue (31 vs 14\%), and infections (23 vs 6\%), predominantly of the upper respiratory tract. The most common grade 3 or 4 drug-related adverse events were stomatitis (7\%), anemia (6\%), and hyperglycemia (5\%), Thereafter, everolimus was approved for the treatment of progressive pNET in patients with unresectable, locally advanced, or metastatic disease by the FDA based on the RADIANT-3 data (Yao et al., 2011).

Temsirolimus is another potent mTOR inhibitor and binds intracellularly to FK506-binding protein-12 and creates a complex that inhibits protein kinase activity of mTOR. Thus, inhibition of mTOR prevents phosphorylation of key cell cycle control proteins, leading to G1 growth arrest. Duran et al. (2006) in their multicenter phase II study including 37 patients with progressive NET treated with the mTOR inhibitor temsirolimus, reported that only 1 of 15 patients with pNET had an 
objective response, but disease control was provided in $67 \%$ of them, which included stable disease for at least two months. Promising preliminary results of another phase II trial was presented at the 2013 ASCO annual meeting. This study evaluated the activity of temsirolimus (25 mg IV weekly) plus the anti-VEGF monoclonal antibody bevacizumab (10 $\mathrm{mg} / \mathrm{kg}$ every other week) in 55 patients with well or moderately differentiated pNET and progressive disease by RECIST within seven months of study entry. Results indicated that a confirmed partial response was documented in 20 patients $(37 \%)$ and 44 $(80 \%)$ remained progression-free at six months. The authors concluded that the combination of temsirolimus/ bevasizumab has substantial activity and phase III trials of combined VEGF/mTOR inhibition in PNET should be pursued (Hobday et al., 2013).

\section{Tyrosine kinase inhibitors}

pNETs are known to be highly vascular tumors with over-expression of VEGF (a key regulator in angiogenesis) and VEGFR (VEGFR)-2, VEGFR-3, platelet derived growth factor receptors (PDGFR)- $\alpha$ and $-\beta$ and c-kit (Fjallskog et al., 2003; Oberg et al., 2013; Zihang et al., 2013). Sunitinib is an oral multikinase inhibitor that has demonstrated actvity against a range of signaling pathways and growth factors/receptors including VEGFR 1, 2, and 3 as well as PDGFR- $\alpha$ and $-\beta$, c-kit, RET. An initial phase II trial explored the activity of sunitinib (50 mg daily for four of every six weeks) in 109 patients with advanced NETs. In $18 \%$ of 61 patients with pNETs, a partial response was obtained, and $68.2 \%$ had prolonged periods of stable disease. In addition, median time to tumor progression (TTP) and 1-year OS rate was 7.7 months and $81.1 \%$, respectively (Kulke et al., 2008).

A multicenter, international randomized, double-blind, and placebo-controlled phase III trial in 171 patients with progressive low to intermediate grade pNET investigated continuous administration of sunitinib (37.5 mg daily) versus placebo (Raymond et al., 2011). Recruitment was terminated prematurely prior to the first preplanned interim efficacy analysis, because the independent data and safety monitoring committee detected more serious adverse events and deaths with placebo, as well as a significant difference in PFS with sunitinib in an interim analysis. Results showed that median PFS time for patients treated with sunitinib was significantly longer than that of patients receiving placebo (11.4 vs 5.5 months, $\mathrm{p}<0.001)$. The probabilty of PFS rate at six months was $71.3 \%$ in the sunitinib arm and $43.2 \%$ in the placebo arm. The objective RR was $9.3 \%$ in the sunitinib arm versus $0 \%$ in the placebo group. The most common grade 3 or 4 adverse events in the sunitinib group were neutropenia (12\%) and hypertension (10\%). Hand-foot skin reaction and hypertension of any grade were determined in $23 \%$ and $26 \%$ of patients receiving sunitinib, respectively. In spite of the side effects related to sunitinib treatment, no differences in the quality-of-life index with sunitinib were dettected (Raymond et al., 2011). Based on these data, sunitinib was approved by FDA for treatment of progressive, well-differentiated pNET in patients with unresectable, locally advanced, or metastatic disease.

Sorafenib is another orally active TK inhibitor, which targets VEGFR-2, RAF and PDGFR- $\beta$. It was previously evaluated in 43 patients with pNETs (Raymond et al., 2011). A preliminary analysis showed that responses were observed in $9 \%$ of the 41 evaluable patients. A prospective study of 51 patients with advanced NET (29 with pNETs and 22 with carcinoid) explored the activity of pazopanib (800 mg Daily), which targets VEGFR-1, VEGFR-2, VEGFR-3, PDGFR- $\alpha$, and $\beta$, as well as c-kit, on stable doses of octreotide-LAR. In a preliminary report, the RR among patients with well-differentiated pancreatic NETs was $17 \%$ (Phan et al., 2010). Selected phase II and III clinical trials of targeted therapies for patients with advanced pNET are summarized in Table 2.

\section{Anti-secretory treatment}

Symptomatic patients associated with hormone hypersecretion from a well-differentiated pNET should be managed with somatostatin analogs and other agents as appropriate to the specific syndrome. Initial anti-secretory treatment includes diazoxide, which directly inhibits the release of insulin from insulinoma cells in insulinoma, high doses of oral proton pump inhibitors in patients with gastrinoma and Zollinger Ellison syndrome. Furthermore, everolimus may be highly effective in improving glycemic

Table 2. Selected Phase II and III Clinical Trials of Targeted Therapies for Patients with Advanced pNET

\begin{tabular}{|c|c|c|c|c|c|}
\hline References & Study/Setting & Treatment & No. of patients & Response rate, $\%$ & $\begin{array}{c}\text { Median PFS/TTP and } \\
\text { OS, months }\end{array}$ \\
\hline \multicolumn{6}{|l|}{ mTOR inhibitors } \\
\hline Yao et al. & Phase II & $\begin{array}{l}\text { Everolimus } v s \text { everolimus- } \\
\text { octreotide }\end{array}$ & 160 & $9 v s 4$ & PFS, 9.7 vs 17 \\
\hline Yao et al & Phase III & $\begin{array}{l}\text { Everolimus-BSC } v s \\
\text { placebo-BSC }\end{array}$ & 410 & $5 v s 2$ & PFS, $11 v s 4.6$ \\
\hline Duran et al. & Phase II & Temsirolimus & 37 & 7 & PFS, 10.6 \\
\hline Hobday et al. & Phase II & Temsirolimus-BEV & 55 & 37 & NA \\
\hline \multicolumn{6}{|l|}{ TK inhibitors } \\
\hline Kulke et al. & Phase II & Sunitinib & 107 & 18 & PFS, 7.7 \\
\hline Raymond et al. & Phase III & Sunitinib $v s$ placebo & 171 & 9.3 vs 0 & PFS, $11.4 v s 5.5$ \\
\hline Raymond et al. & Phase II & Sorafenib & 43 & 9 & PFS, 11.9 \\
\hline Phan et al. & Phase II & Pazopanib-octreotide & 31 & 17 & 11-Jul \\
\hline
\end{tabular}

pNET, pancreatic neuroendocrine tumor; BSC, best supportive care; PFS, Progression-free survival; TTP,Time-to progression; OS, overall survival, NA, not applicable 
control in patients with insulinoma. In refractory cases with gastrinoma, somatostatin analogs may be helpful (Hirshberg et al., 2005; Norton et al., 2005).

\section{Somatostatin analogs}

There are at least five subtypes or SSTRs (sst15), a G-protein-coupled membrane receptor to which native somatostatin peptides bind with high affinity. Aproximately $80 \%$ of NETs express somatostatin receptors (SSTRs), predoninantly sst-5, but in only half of insulinomas and less in poorly differentiated NETs (Baldelli et al., 2014). Somatostatin is a 14-amino acid peptide that inhibits the secretion of a broad range of hormones in vivo. Somatostatin analogs act by binding to somatostatin receptors, so effectively inhibit hormonal secretion and ameliorate symptoms such as flushing and diarrhea (O'Toole et al., 2000; Baldelli et al., 2014; Yucel et al., 2014). There are currently two somatostatin analogs, octreotide and lanreotide, and they bind mainly sst- 2 and sst-5 with high affinity. Although both drugs have a longer half-life than endogenous somatostatin, depot preperations have been developed (ocreaotide LAR and lanreotide MP and AG) (Baldelli et al., 2014). A prospective crossover study showed that there were no differences with respect to symptom control or biochemical response between the two drugs (O'Toole et al., 2000).

The activity of somatostatin analogs has been previously explored in the treatment of pNET and confirmed by multiple clinical trials. In addition, several studies have indicated that about two-thirds of patients with NETs who received octreotide experienced stable disease for up to five years, while objective response was seen in only 5\% of patients (Aparicio et al., 2001; Shojamanesh et al., 2002; Panzuto et al., 2006). It was thought that there might be direct antitumor effects by stimulation of sst-2, which results in cell-cycle arrest and apoptosis. Afterythat, a first double-blind, placebocontrolled, and randomized phase III PROMID study was performed by Rinke et al., evaluating the antitumor activity of octreotide LAR, and involving 85 patients with well-differentiated metastatic midgut NETs (Rinke et al., 2009). The authors showed that the median TTP time for patients who received ocreotide LAR $30 \mathrm{mg}$ monthly was significantly better than that of patients treated with placebo (14.3 vs 6.0 months, $\mathrm{p}=0.000072$ ). After six months of treatment, stable disease was obtained in $66.7 \%$ of patients receiving octreotide LAR compared with $37.2 \%$ of placebo recipients. Long-term survival results were presented at the ASCO 2013 annual meeting, but OS was found to be significantly different at 84 months ( $p=0.59)$ (Arnold et al., 2013).

Lanreotide is another of the long-lasting somatostatin analogs, which binds to SSTR with a similar profile like octreotide. Recently, the pahse III CLARINET trial was published and provided further support for the antiproliferative effect of somatostatin analogs. This trial compared lanreotide with placebo in 204 patients with metastatic or locally advanced well- or moderately differentiated, non-functioning pNETs or intestinal NETs (Caplin et al., 2014). Patients were randomly assigned to receive an extended-release Autogel formulation of lanreotide at a dose of $120 \mathrm{mg}$ (101 patients) or placebo (103 patients) once every 28 days for 96 weeks or until progressive disease or death. Most patients (96\%) had no tumor progression in the three to six months before randomization; approximately one-half had pancreatic primary sites. Results indicated that lanreotide, as compared with placebo, was associated with significantly prolonged PFS (median not reached vs 18.0 months, respectively, $\mathrm{p}<0.001$, hazard ratio for progression or death, 0.47). The estimated rates of PFS at 24 months were $65.1 \%$ in the lanreotide group and $33.0 \%$ in the placebo group. There were no significant differences in quality of life or OS. The most common treatment-related adverse effect was diarrhea (26\% versus $9 \%$ in the lanreotide and placebo groups, respectively).

Pasireotide (SOM230) is a new multireceptortargeted somatostatin analog and binds four of the five SSTRs (sst1,2,3,5) with high affinity. It may benefit a wider spectrum of patients with functional NETs who have not responded to prior treatment with octreotide or lanreotide, because it has a 40 -fold higher affinity and 158-fold higher functional activity for sst-5 compared with octreotide (Schmid et al., 2008). A phase II study showed effective control of diarrhea and flushing in patients with NETs who were refrectory or resistant to octreotide LAR (Kvols et al., 2006). Several phase II trials are currently underway or planned including pasireotide alone or plus everolimus in patients with pNET or NETs (NCT01253161, NCT01374451, NCT00088595). Table 3 shows selected trials of somatostatine analogues in patients with advanced pNET.

\section{Interferon therapy}

The anti-secretory and anti-proliferative effects of INF- $\alpha$ have been previously investigated as monotherapy

Table 3. Selected Trials of Somatostatine Analogues in Patients with Advanced pNET

\begin{tabular}{llclc}
\hline References & \multicolumn{1}{c}{ Regimen } & No. of patients & Response rate, $\%$ & $\begin{array}{c}\text { Median PFS/TTP and } \\
\text { OS, months }\end{array}$ \\
\hline Panzuto et al. & Oct LAR 30 mg q28day & 21 & SD:45 & NA \\
Shojamanesh et al. & Oct sc or OCT LAR & 15 & PR:4; SD:28 & NA \\
Aparicio et al. & Oct sc 100 $\mu$ g t.i.d. or Lan i.m 30 mg & 35 & PR:2.9; SD:57.1 & NA \\
& q14 day to q7 day or both & & & PFS, $14.3 v s 6.0$ \\
Rinke et al. & Oct LAR 30 mg q28 day $v s$ placebo & 85 & SD:66.7 vs 37.2 & PFS, NR $v s$ 18 \\
Caplin et al. & Lan 120 mg q28 day $v$ placebo & 204 & NA
\end{tabular}

**pNET: pancreatic neuroendocrine tumors, Oct: octreotide; Lan: lanreotide; BSC: Best supportive care; sc: subcutaneous; i.m: intramuscular;NA: not applicable; PFS: progression-free survival; OS: overall survival; TTP: time-to progression, SD:stable disease; PR: partial response; NR: not reached 
or with somatostatin analogs (Oberg, 1992; Fjallskog et al., 2002; Arnold et al., 2005). Oberg et al. (2005) showed that symptomatic and biochemical responses were observed in $50 \%$ of patients with disease stabilization at a follow-up of four years. On the other hand, significant tumor shrinkage was evident in only $10-15 \%$. The benefit of INF- $\alpha$ in combination with octreotide with respect to survival could not be proved in patients with NETs by a few studies (Fjallskog et al., 2002; Arnold et al., 2005). Some side effects that include flu-like symptoms, bone morrow supression, and chronic fatigue may limit the use of INF- $\alpha$, but the tolerability of low-dose regimens has been demonstrated in clinical practice.

\section{Liver-directed treatments}

Liver-directed treatments may be used in patients with progressive liver-predominant metastatic disease. These approaches may reduce tumor bulk and ameliorate symptoms due to hormone secretion (Forrer et al., 2007). Desai et al. (2001) in their study of chemoembolization combined with somatostatin therapy indicated that the relief of symptoms was observed in $78 \%$ of patients. In addtion, radiographic improvement or stability was seen in 18 patients $(45 \%)$. In a study of radioembolization using resin 90Y-microspheres including 148 patients, a complete response was seen in $2.7 \%$, partial response in $60.5 \%$, stable disease in $22.7 \%$, and progressive disease in $4.5 \%$. No radiation liver failure occurred and median survival was 70 months (Kennedy et al., 2008).

\section{Peptide-receptor radionuclide therapy (PRRT)}

Another liver-directed option for patients is the use of radio labeled somatostatin analogs and has also been studied. Patients with non-resectable metastases and positive octreoscan may be used for PRRT. However, randomized controlled phase III trials are lacking and experience is limited to single-institution series. The predominant isotopes used in PRRT are $177 \mathrm{Lu}$ and $90 \mathrm{Y}$ and RR was reported to be 10-30\% (Kwekkeboom et al., 2008; Sowa-Staszczak et al., 2011; Villard et al., 2012; Gulenchyn et al., 2012). Kwekkeboom et al. (2008) reported that there was complete response in $2 \%$, partial response in $28 \%$, and minor responses in $16 \%$ in 310 patients treated with the analog $177 \mathrm{Lu}-\mathrm{DOTA}$ 0, TYR3 octreotate (177Lu-DOTATATE). Moreover, the median TTP was 40 months, and toxicity was minor. For non-functional pNET, a partial RR was reportted to be $36 \%$ in this trial. Toxicity is an important problem for PRRT approach, because of myelosuppression and therapy-related myelodysplastic syndrome (Villard et al., 2012; Gulenchyn et al., 2012). Therefore, PRRT may be considered for patients with metaiodobenzylguanidine (MIBG)/octreotide avid disease, good performance status, adequate organ fuction, and low bulk disease.

\section{Future Directions and Ongoing Trials}

In recent years, tremendous advancements have been made in the treatment of patients with pNETs, especially because the activity of novel targeted agents, such as sunitinib and everolimus, has been proved, and new trials have focused on the cellular mechanism of growth and tumor maintenance in pNETs. Further phase I and II with respect to the targeted treatments are currently underway or planned including cabozantinib (oral multikinase inhibitor), cixutumumab, AMG479 (anti-IGF1R monoclonal antibodies), pazopanib monotherapy and pazopanib plus temozolamide, and dovitinib in metastatic or unresectable pNETs (NCT01466036, NCT01204476, NCT01024387, NCT01280201, NCT01465659, NCT02108782).

In addition, phase III RADIANT-4 study is ongoing. The purpose of this study is to compare the antitumor activity of everolimus plus BSC with placebo plus BSC in patients with advanced nonfunctional NETs of gastrointestinal or lung origin (NCT01524783). A study evaluating lanreotide as maintenance therapy in patients with unresectable duodeno-pNETs is also underway (NCT02288377). The inhibition of a single signaling pathway in pNETs tumor cells may be posed the development of resistant and escape mechanisms. Therefore, further treatments have been aimed to determine the activity of combination of targeted therapy in combination with the other targeted agents or liverdirected therapies, or chemotherapy. Capecitabine and temozolomide in combination anti-VEGF, bevacizumab for metastatic or unresectable pNETs are currently being explored in phase II study (NCT01525082). Furthermore, another phase II trial is evaluating the activity of everolimus and octreotide with or without bevacizumab in locally advanced or metastatic pNETs (NCT01229943).

There have been no studies to compare the different types of treatment. Since the patient is eligible for treatment with either sunitinib, everolimus or cytotoxic chemotherapy, some molecular markers may help in determining future patients' benefits from these therapies. Moreover, there are no definitively proven predictive biomarkers to evaluate response to targeted therapies in patients with pNET. Currently, a study evaluating potential imaging and molecular predictors of response to novel therapies in metastatic pNETs is ongoing (NCT01603004).

\section{Conclusions}

pNETs are heterogenous tumors and relatively rare compared with the other cancer subtypes. Therefore, the planning of large randomized studies with respect to the novel therapies is difficult. Recently, there has been significant advance in the treatment of patients with pNETs with the advent of new small, orally active tageted drugs such as sunitinib and everolimus. In addition, several studies evaluating the activity of other targeted agents alone or in combination with the other targeted drugs or chemotherapy are currently ongoing. In spite of this significant progress, there is an unmet need for further insights into new systemic treatment approaches, development of novel targeted agents, molecular tumorigenesis, and prognostic or predictive biomarkers in patients with pNETs. 


\section{References}

Aparicio T, Ducreux M, Baudin E, et al (2001). Antitumour activity of somatostatin analogues in progressive metastatic neuroendocrine tumours. Eur J Cancer, 37, 1014-9.

Arnold R, Rinke A, Klose KJ, et al (2005). Octreotide versus octreotide plus interferon-alpha in endocrine gastroenteropancreatic tumors: a randomized trial. Clin Gastroenterol Hepatol, 3, 761-71.

Arnold R, Wittenberg M, Rinke A, et al (2013). Placebo controlled, double blind, prospective, randomized study on the effect of octreotide LAR in the control of tumor growth in patients with metastatic neuroendocrine midgut tumors (PROMID): Results on long-term survival. J Clin Oncol, 31, abstract 4030.

Bajetta E, Catena L, Procopio G, et al (2007). Are capecitabine and oxaliplatin (XELOX) suitable treatments for progressing low-grade and high-grade neuroendocrine tumours? Cancer Chemother Pharmacol, 59, 637-42.

Baldelli R, Barnabei A, Rizza L, et al (2014). Somatostatin analogs therapy in gastroenteropancreatic neuroendocrine tumors: current aspects and new perspectives. Front Endocrinol (Lausanne), 5, 7.

Ballian N, Loeffler AG, Rajamanickam V, et al (2009). A simplified prognostic system for resected pancreatic neuroendocrine neoplasms. HPB (Oxford), 11, 422-8.

Chan JA, Stuart K, Earle CC, et al (2012). Prospective study of bevacizumab plus temozolomide in patients with advanced neuroendocrine tumors. J Clin Oncol, 30, 2963-8.

Chan JA, Blaszkowsky L, Stuart K, et al (2013). A prospective, phase $1 / 2$ study of everolimus and temozolomide in patients with advanced pancreatic neuroendocrine tumor. Cancer, 119, 3212-8

Caplin ME, Pavel M, Cwikla JB, et al (2014). Lanreotide in metastatic enteropancreatic neuroendocrine tumors. $N \mathrm{Engl}$ J Med, 371, 224-33.

Castellano D, Capdevila J, Sastre J, et al (2013). Sorafenib and bevacizumab combination targeted therapy in advanced neuroendocrine tumour: a phase II study of Spanish neuroendocrine tumour group (GETNE0801). Eur J Cancer, 49, 3780-7

Delaunoit T, Ducreux M, Boige V, et al (2004). The doxorubicinstreptozotocin combination for the treatment of advanced well-differentiated pancreatic endocrine carcinoma; a judicious option? Eur J Cancer, 40, 515-20.

Desai DC, O'Dorisio TM, Schirmer WJ, et al (2001). Serum pancreastatin levels predict response to hepatic artery chemoembolization and somatostatin analogue therapy in metastatic neuroendocrine tumors. Regul Pept, 96, 113-7.

Duran I, Kortmansky J, Singh D, et al (2006). A phase II clinical and pharmacodynamic study of temsirolimus in advanced neuroendocrine carcinomas. Br J Cancer, 95, 1148-54.

Eriksson B, Skogseid B, Lundqvist G, et al (1990). Medical treatment and long-term survival in a prospective study of 84 patients with endocrine pancreatic tumors. Cancer, $\mathbf{6 5}$, $1883-90$.

Fjällskog ML, Lejonklou MH, Oberg KE, Eriksson BK, Janson ET (2003). Expression of molecular targets for tyrosine kinase receptor antagonists in malignant endocrine pancreatic tumors. Clin Cancer Res, 9, 1469-73.

Fjällskog ML, Sundin A, Westlin JE, et al (2002). Treatment of malignant endocrine pancreatic tumors with a combination of alpha-interferon and somatostatin analogs. Med Oncol, 19, 35-42.

Fine RL, Gulati AP, Tsushima D, et al (2014). Prospective phase II study of capecitabine and temozolomide (CAPTEM) for progressive, moderately, and well-differentiated metastatic neuroendocrine tumors. J Clin Oncol, 32, abstract 179.

Forrer F, Valkema R, Kwekkeboom DJ, de Jong M, Krenning EP (2007). Neuroendocrine tumors. peptide receptor radionuclide therapy. Best Pract Res Clin Endocrinol Metab, 21, 111-29.

Gulenchyn KY, Yao X, Asa SL, Singh S, Law C (2012). Radionuclide therapy in neuroendocrine tumours: a systematic review. Clin Oncol (R Coll Radiol), 24, 294-308.

Hainsworth JD, Spigel DR, Litchy S, Greco FA (2006). Phase II trial of paclitaxel, carboplatin, and etoposide in advanced poorly differentiated neuroendocrine carcinoma: a minnie pearl cancer research network Study. J Clin Oncol, 24, 3548-54.

Halfdanarson TR, Rubin J, Farnell MB, Grant CS, Petersen GM (2008). Pancreatic endocrine neoplasms: epidemiology and prognosis of pancreatic endocrine tumors. Endocr Relat Cancer, 15, 409-27.

Hirshberg B, Cochran C, Skarulis MC, et al (2005). Malignant insulinoma: spectrum of unusual clinical features. Cancer, 104, 264-72.

Hobday TJ, Qin R, Moore MJ, et al (2013). Multicenter phase II trial of temsirolimus (TEM) and bevacizumab (BEV) in pancreatic neuroendocrine tumor (PNET). J Clin Oncol, 31, abstract 4032.

Jensen RT, Berna MJ, Bingham DB, Norton JA (2008). Inherited pancreatic endocrine tumor syndromes: advances in molecular pathogenesis, diagnosis, management, and controversies. Cancer, 113, 1807-43.

Khagi S, Saif MW (2015). Pancreatic neuroendocrine tumors: targeting the molecular basis of disease. Curr Opin Oncol, 27, 38-43.

Kennedy AS, Dezarn WA, McNeillie P, et al (2008). Radioembolization for unresectable neuroendocrine hepatic metastases using resin 90Y-microspheres: early results in 148 patients. Am J Clin Oncol, 31, 271-9.

Knigge U, Hansen CP, Stadil F (2008). Interventional treatment of neuroendocrine liver metastases. Surgeon, 6, 232-9.

Kouvaraki MA, Ajani JA, Hoff P, et al (2004). Fluorouracil, doxorubicin, and streptozocin in the treatment of patients with locally advanced and metastatic pancreatic endocrine carcinomas. J Clin Oncol, 22, 4762-71.

Kulke MH, Hornick JL, Frauenhoffer C, et al (2009). O6methylguanine DNA methyltransferase deficiency and response to temozolomide-based therapy in patients with neuroendocrine tumors. Clin Cancer Res, 15, 338-45.

Kunz PL, Kuo T, Zahn JM, et al (2010). A phase II study of capecitabine, oxaliplatin, and bevacizumab for metastatic or unresectable neuroendocrine tumors. J Clin Oncol, 28, abstract 4104.

Kulke MH, Lenz HJ, Meropol NJ, et al (2008). Activity of sunitinib in patients with advanced neuroendocrine tumors. J Clin Oncol, 26, 3403-10.

Kulke MH, Stuart K, Enzinger PC, et al (2006). Phase II study of temozolomide and thalidomide in patients with metastatic neuroendocrine tumors. J Clin Oncol, 24, 401-6.

Kwekkeboom DJ, de Herder WW, Kam BL, et al (2008). Treatment with the radiolabeled somatostatin analog [177 Lu-DOTA 0,Tyr3]octreotate: toxicity, efficacy, and survival. $J$ Clin Oncol, 26, 2124-30.

Kvols L, Wiedenmann B, Oberg K, et al (2006). The SOM230 carcinoid study group. safety and efficacy of pasireotide (SOM230) in patients with metastatic carcinoid tumors refractory or resistant to octreotide LAR: results of a phase II study. J Clin Oncol, 24, abstract 4082.

Missiaglia E, Dalai I, Barbi S, et al (2010). Pancreatic endocrine tumors: expression profiling evidences a role for AKTmTOR pathway. J Clin Oncol, 28, 245-55. 
Mitry E, Baudin E, Ducreux M, et al (1999). Treatment of poorly differentiated neuroendocrine tumours with etoposide and cisplatin. Br J Cancer, 81, 1351-5.

Modlin IM, Pavel M, Kidd M, Gustafsson BI (2010). Review article: somatostatin analogues in the treatment of gastroenteropancreatic neuroendocrine (carcinoid) tumours. Aliment Pharmacol Ther, 31, 169-88.

Moertel CG, Lefkopoulo M, Lipsitz S, Hahn RG, Klaassen D (1992). Streptozocin-doxorubicin, streptozocin-fluorouracil or chlorozotocin in the treatment of advanced islet-cell carcinoma. N Engl J Med, 326, 519-23.

Niederle MB, Hackl M, Kaserer K, Niederle B (2010). Gastroenteropancreatic neuroendocrine tumours: the current incidence and staging based on the WHO and European Neuroendocrine Tumour Society classification: an analysis based on prospectively collected parameters. Endocr Relat Cancer, 17, 909-18.

Norton JA, Alexander HR, Fraker DL, et al (2003). Possible primary lymph node gastrinoma: occurrence, natural history, and predictive factors: a prospective study. Ann Surg, 237, 650-7.

Oberg K (1992). Interferons in the management of neuroendocrine tumors and their possible mechanism of action. Yale J Biol Med, 65, 519-29.

Oberg K (2000). Interferon in the management of neuroendocrine GEP-tumors: a review. Digestion, 62, 92-7.

Oberg K, Casanovas O, Castano JP, et al (2013). Molecular pathogenesis of neuroendocrine tumors: implications for current and future therapeutic approaches. Clin Cancer Res, 19, 2842-9.

Olsen IH, Langer SW, Jepsen I, et al (2012). First-line treatment of patients with disseminated poorly differentiated neuroendocrine carcinomas with carboplatin, etoposide, and vincristine: a single institution experience. Acta Oncol, 51, 97-100.

O’Toole D, Ducreux M, Bommelaer G, et al (2000). Treatment of carcinoid syndrome: a prospective crossover evaluation of lanreotide versus octreotide in terms of efficacy, patient acceptability, and tolerance. Cancer, 88, 770-6.

Pavel M, Baudin E, Couvelard A, et al (2012); Barcelona Consensus Conference participants. ENETS Consensus Guidelines for the management of patients with liver and other distant metastases from neuroendocrine neoplasms of foregut, midgut, hindgut, and unknown primary. Neuroendocrinology, 95, 157-76.

Phan, AT, Yao, JC, Fogelman, DR, et al (2010). A prospective, multi-institutional phase II study of GW786034 (pazopanib) and depot octreotide (sandostatin LAR) in advanced lowgrade neuroendocrine carcinoma (LGNEC). J Clin Oncol, 28, abstract 4001.

Panzuto F, Di Fonzo M, Iannicelli E, et al (2006). Long-term clinical outcome of somatostatin analogues for treatment of progressive, metastatic, well-differentiated entero-pancreatic endocrine carcinoma. Ann Oncol, 17, 461-6.

Ramage JK, Ahmed A, Ardill J, et al (2012). UK and Ireland neuroendocrine tumour society. guidelines for the management of gastroenteropancreatic neuroendocrine (including carcinoid) tumours (NETs). Gut, 61, 6-32.

Ramanathan RK, Cnaan A, Hahn RG, Carbone PP, Haller DG (2001). Phase II trial of dacarbazine (DTIC) in advanced pancreatic islet cell carcinoma. Study of the Eastern cooperative oncology group-E6282. Ann Oncol, 12, 1139-43.

Raymond E, Dahan L, Raoul JL, et al (2011). Sunitinib malate for the treatment of pancreatic neuroendocrine tumors. $N$ Engl J Med, 364, 501-13.

Raymond E, Hobday T, Castellano D, et al (2011). Therapy innovations: tyrosine kinase inhibitors for the treatment of pancreatic neuroendocrine tumors. Cancer Metastasis Rev, 30, 19-26.

Rinke A, Muller HH, Schade-Brittinger C, et al (2009). Placebocontrolled, double-blind, prospective, randomized study on the effect of octreotide LAR in the control of tumor growth in patients with metastatic neuroendocrine midgut tumors: a report from the PROMID study group. J Clin Oncol, 27, 4656-63.

Schmid HA (2008). Pasireotide (SOM230): development, mechanism of action and potential applications. Mol Cell Endocrinol, 286, 69-74.

Strosberg JR, Nasir A, Hodul P, Kvols L (2008). Biology and treatment of metastatic gastrointestinal neuroendocrine tumors. Gastrointest Cancer Res, 2, 113-25.

Singh S, Dey C, Kennecke H, et al (2014) Consensus recommendations for the diagnosis and management of pancreatic neuroendocrine tumors: Guidelines from a Canadian national expert group. Ann Surg Oncol, [Epub ahead of print].

Shojamanesh H, Gibril F, Louie A, et al (2002). Prospective study of the antitumor efficacy of long-term octreotide treatment in patients with progressive metastatic gastrinoma. Cancer, 94, 331-43.

Sowa-Staszczak A, Pach D, Stefanska A, et al (2011). Can treatment using radiolabelled somatostatin analogue increase the survival rate in patients with non-functioning neuroendocrine pancreatic tumours? Nucl Med Rev Cent East Eur, 14, 73-8.

Strosberg JR, Fine RL, Choi J, et al (2011). First-line chemotherapy with capecitabine and temozolomide in patients with metastatic pancreatic endocrine carcinomas. Cancer, 117, 268-75.

Sun W, Lipsitz S, Catalano P, Mailliard JA, Haller DG (2005). Phase II/III study of doxorubicin with fluorouracil compared with streptozocin with fluorouracil or dacarbazine in the treatment of advanced carcinoid tumors: Eastern cooperative oncology group study E1281. J Clin Oncol, 23, 4897-904.

Tokunaga C, Yoshino K, Yonezawa K (2004). mTOR integrates amino acid- and energy-sensing pathways. Biochem Biophys Res Commun, 313, 443-6.

Venook AP, Ko AH, Ko AH, et al (2008). Phase II trial of FOLFOX plus bevacizumab in advanced, progressive neuroendocrine tumors. J Clin Oncol, 26, abstract 216.

Villard L, Romer A, Marincek N, et al (2012). Cohort study of somatostatin-based radiopeptide therapy with [(90) Y-DOTA]-TOC versus [(90)Y-DOTA]-TOC plus [(177) Lu-DOTA]-TOC in neuroendocrine cancers. J Clin Oncol, 30, 1100-6.

Yao JC, Phan A, Hoff PM, et al (2008). Targeting vascular endothelial growth factor in advanced carcinoid tumor: a random assignment phase II study of depot octreotide with bevacizumab and pegylated interferon alpha-2b. J Clin Oncol, 26, 1316-23.

Yao JC, Lombard-Bohas C, Baudin E, et al (2010). Daily oral everolimus activity in patients with metastatic pancreatic neuroendocrine tumors after failure of cytotoxic chemotherapy: a phase II trial. J Clin Oncol, 28, 69-76.

Yao JC, Hassan M, Phan A, et al (2008). One hundred years after "carcinoid": epidemiology of and prognostic factors for neuroendocrine tumors in 35,825 cases in the United States. J Clin Oncol, 26, 3063-72.

Yao JC, Shah MH, Ito T, et al (2011). RAD001 in advanced neuroendocrine tumors, third trial (RADIANT-3) study group. everolimus for advanced pancreatic neuroendocrine tumors. N Engl J Med, 364, 514-23.

Yao KA, Talamonti MS, Nemcek A, et al (2001). Indications and results of liver resection and hepatic chemoembolization for 
metastatic gastrointestinal neuroendocrine tumors. Surgery,

130, 677-82.

Yucel B, Babacan NA, Kacan T, et al (2014). Survival analysis and prognostic factors for neuroendocrine tumors in Turkey. Asian Pac J Cancer Prev, 14, 6687-92.

Zeng YJ, Liu L, Wu H, et al (2013). Clinicopathological features and prognosis of gastroenteropancreatic neuroendocrine tumors: analysis from a single-institution. Asian Pac J Cancer Prev, 14, 5775-81.

Zhang J, Francois R, Iyer R, et al (2013). Current understanding of the molecular biology of pancreatic neuroendocrine tumors. J Natl Cancer Inst, 105, 1005-17.

Zitzmann K, De Toni EN, Brand S, et al (2007). The novel mTOR inhibitor RAD001 (everolimus) induces antiproliferative effects in human pancreatic neuroendocrine tumor cells. Neuroendocrinology, 85, 54-60. 